Bulletin of the Section of Logic

Volume 47/3 (2018), pp. 159-170

http://dx.doi.org/10.18778/0138-0680.47.3.02

Zofia Kostrzycka

\title{
ON INTERPOLATION IN NEXT(KB.Alt(2))
}

\begin{abstract}
We prove that there is infinitely many tabular modal logics extending KB.Alt(2) which have interpolation.
\end{abstract}

Keywords: symmetric Kripke frames, interpolation, amalgamation.

\section{Introduction}

There is a huge literature on interpolation in modal logics, see [1], pp. 443470. The best known is the result by Maksimova [6] who showed that there is finitely many normal extensions of $\mathbf{S} \mathbf{4}$ enjoying interpolation. Despite this, little is known about interpolation in intransitive modal logics (that are not extensions of $\mathbf{S 4}$ ). We try to fill the gap. We prove that there exists infinitely many logics without interpolation both in $N E X T\left(\mathbf{K T B} .4_{2}\right)$ and $N E X T($ KTB.Alt(3)) see [4], [5] appropriately.

In this paper we study some normal extensions of the modal logic $\mathbf{K B}:=\mathbf{K} \oplus B$, where $B:=p \rightarrow \square \diamond p$. The logic $\mathbf{K B}$ is complete with respect to the class of symmetric Kripke frames.

The paper is a continuations of [4] and [5] concerning the interpolation in $N E X T(\mathbf{K T B})$. In [4] an infinite family of logics without interpolation is described. The logics studied there are weakly transitive and belong to $N E X T\left(\mathbf{K T B} .4_{2}\right)$. In [5] some, mostly negative, results concerning interpolation for intransitive logics are included. The logics considered there are characterized by reflexive, symmetric and linear Kripke frames. We considered extensions of KTB.Alt $(\mathbf{3}):=\mathbf{K} \oplus T \oplus B \oplus$ alt $_{3}$, with

$$
\begin{aligned}
& T:=\square p \rightarrow p \\
& \text { alt }_{3}:=\square p \vee \square(p \rightarrow q) \vee \square((p \wedge q) \rightarrow r) \vee \square((p \wedge q \wedge r) \rightarrow s) .
\end{aligned}
$$


The axiom $\left(a l t_{3}\right)$ is a special case of a more general $\left(a l t_{n}\right), n \geq 1$ :

$$
\text { alt }_{n}:=\square p_{1} \vee \square\left(p_{1} \rightarrow p_{2}\right) \vee \ldots \vee \square\left(\left(p_{1} \wedge \ldots \wedge p_{n}\right) \rightarrow p_{n+1}\right) .
$$

It is proven in [5] that the logic KTB.Alt(3) does not have interpolation and it has only two normal extensions having this property. These are the following: $L(\circ)=$ Triv and $L(\circ--\circ)-$ logics determined by one-point or two-point cluster, appropriately. One may notice that the situation is analogous to that of S5, see [1], p. 463.

In this paper we contrast the negative results from [5] for $N E X T($ KTB.Alt $(3))$ with positive ones for $N E X T($ KB.Alt $(2))$. The common feature of the Kripke frames for both the families is their linearity. However, in the former case the appropriate Kripke frames are chains of reflexive points, whereas in the latter one, they are chains of (possibly) irreflexive points.

\section{Preliminaries}

Let us recall the basic definitions. The symbol $\operatorname{Var}(\alpha)$ means the set of all propositional variables in the formula $\alpha$.

Definition 1. A logic L has the Craig interpolation property (CIP) if for every implication $\alpha \rightarrow \beta$ in $L$, there exists a formula $\gamma$ (interpolant for $\alpha \rightarrow \beta$ in $L)$ such that

$$
\alpha \rightarrow \gamma \in L \text { and } \gamma \rightarrow \beta \in L
$$

and $\operatorname{Var}(\gamma) \subseteq \operatorname{Var}(\alpha) \cap \operatorname{Var}(\beta)$.

DeFinition 2. A logic L has interpolation for deducibility (IPD) if for any $\alpha$ and $\beta$ the condition $\alpha \vdash_{L} \beta$ implies that there exists a formula $\gamma$ such that

$$
\alpha \vdash_{L} \gamma \text { and } \gamma \vdash_{L} \beta
$$

and $\operatorname{Var}(\gamma) \subseteq \operatorname{Var}(\alpha) \cap \operatorname{Var}(\beta)$.

It is well known that (CIP) together with (MP) and deduction theorem implies (IPD). It is known that $\mathbf{K}, \mathbf{T}, \mathbf{K} \mathbf{4}$ and $\mathbf{S 4}$ have (CIP), see Gabbay [3]. Also the logics from $N E X T(\mathbf{S} 4)$ are well recognized as regards interpolation (see [6], also [1], pp. 462-463). In particular, S5 has (CIP). The last fact can be proven by applying a very general method of construction of inseparable tableaux (see i.e. [1], pp. 446-449). The same method 
can be applied in the case of $\mathbf{K T B}$ and $\mathbf{K B}$. Therefore, without getting into details, we conclude that both the logics KTB and KB have (CIP). The method of construction of inseparable tableaux is not applicable for the logics KTB.Alt(n) and KB.Alt(n), $n \geq 2$. In [5] it is proven that KTB.Alt(3) does not have (CIP).

In the next section we shall look for tabular logics from $N E X T(\mathbf{K B}$.Alt $(\mathbf{2}))$ having (IPD) or (CIP). Our approach is purely semantic. We shall consider logics determined by classes of Kripke frames $\mathcal{K}$. Formally, the logic determined by a class $\mathcal{K}$ is defined as follows:

$$
L(\mathcal{K}):=\{\alpha \in \text { Form }: \mathfrak{F} \models \alpha \text { for each } \mathfrak{F} \in \mathcal{K}\} .
$$

Note that the class $\mathcal{K}$ may consist of one frame only. Hereafter symmetric Kripke frames will be called $K B$-frames. Symmetric and linear Kripke frames are said to be $K B$.Alt(2)-frames.

The properties (CIP) and (IPD) have an appropriate algebraic characterization, (see [6], [2]). The symbol $V(L)$ denotes the variety of modal algebras characterizing the logic $L$. From [6] the following equivalence is known for any logic $L \in N E X T(\mathbf{K})$ : $L$ possesses (CIP) iff $V(L)$ has the superamalgamation property. In [2] it is shown that $L$ possesses (IPD) iff $V(L)$ has the amalgamation property.

By theory of duality between Kripke frames and modal algebras, amalgamation and superamalgamation properties are transformed into appropriate properties for classes $\mathcal{K}$ of Kripke frames. We need to recall the notion of p-morphism, first.

DeFinition 3. Let $\mathfrak{F}_{1}:=\left\langle W_{1}, R_{1}\right\rangle$ and $\mathfrak{F}_{2}:=\left\langle W_{2}, R_{2}\right\rangle$ be Kripke frames. A map $f: W_{1} \rightarrow W_{2}$ is a p-morphism from $\mathfrak{F}_{1}$ to $\mathfrak{F}_{2}$, if it satisfies the following conditions:

(p1) $f$ maps $W_{1}$ onto $W_{2}$,

(p2) for all $x, y \in W_{1}, x R_{1} y$ implies $f(x) R_{2} f(y)$,

(p3) for each $x \in W_{1}$ and for each $a \in W_{2}$, if $f(x) R_{2} a$ then

there exists $y \in W_{1}$ such that $x R_{1} y$ and $f(y)=a$.

It is said also that the frame $\mathfrak{F}_{1}$ is reducible to $\mathfrak{F}_{2}$ or that the frame $\mathfrak{F}_{2}$ is a p-morphic reduct of $\mathfrak{F}_{1}$.

The next lemma is a folklore in logic: 
LEMMA 1. Let $\mathfrak{F}_{1}, \mathfrak{F}_{2}$ be Kripke frames. If there exists a p-morphism from $\mathfrak{F}_{1}$ to $\mathfrak{F}_{2}$ then $L\left(\mathfrak{F}_{1}\right) \subseteq L\left(\mathfrak{F}_{2}\right)$.

For some special Kripke frames Lemma 1 may be strengthened to an equivalence. The Jónsson's lemma, the congruence extension property of modal algebras, finiteness and simplicity of the dual algebra for $\mathfrak{F}_{1}$ are exploited. The proof is analogous to the one from [7]. We get as follows:

Lemma 2. Let $\mathfrak{F}_{1}, \mathfrak{F}_{2}$ be finite and connected $K B$-frames. Then $L\left(\mathfrak{F}_{1}\right) \subseteq$ $L\left(\mathfrak{F}_{2}\right)$ iff there exists a p-morphism from $\mathfrak{F}_{1}$ onto $\mathfrak{F}_{2}$.

Then we get the amalgamation property for class of frames (APK)

DeFinition 4. For any $\mathfrak{F}_{0}, \mathfrak{F}_{1}$ and $\mathfrak{F}_{2}$ in class $\mathcal{K}$ and for any $p$-morphism $f_{1}: \mathfrak{F}_{1} \rightarrow \mathfrak{F}_{0}$ and $f_{2}: \mathfrak{F}_{2} \rightarrow \mathfrak{F}_{0}$ there exist $\mathfrak{F}$ in $\mathcal{K}$ and $p$-morphisms $g_{1}: \mathfrak{F} \rightarrow \mathfrak{F}_{1}$ and $g_{2}: \mathfrak{F} \rightarrow \mathfrak{F}_{2}$ such that $f_{1} \circ g_{1}=f_{2} \circ g_{2}$. See Figure 1 .

Superamalgamation property for frames except (APK) requires the additional condition (SAPK):

$$
\forall_{x \in \mathfrak{F}_{1}} \forall_{y \in \mathfrak{F}_{2}}\left[f_{1}(x)=f_{2}(y) \Rightarrow \exists_{z \in \mathfrak{F}} g_{1}(z)=x \wedge g_{2}(z)=y\right] .
$$

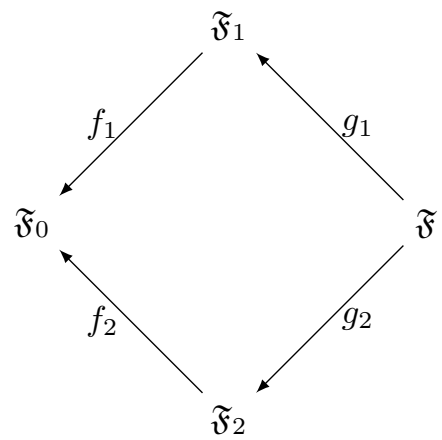

Fig. 1 .

Dealing with logics determined by classes of finite frames we have the following equivalence.

TheOREM 1. Let $L \in N E X T(\mathbf{K B}), L$ be determined by a class $\mathcal{K}$ of finite Kripke frames and $\mathcal{K}$ be closed under p-morphisms. For the logic $L$ the following is equivalent: 
- $L$ possesses (CIP),

- $\mathcal{K}$ has the superamalgamation property (SAPK).

Theorem 2. Let $L \in N E X T(\mathbf{K B}), L$ be determined by a class $\mathcal{K}$ of finite Kripke frames and $\mathcal{K}$ be closed under p-morphisms. For the logic $L$ the following is equivalent:

- $L$ possesses (IPD),

- $\mathcal{K}$ has the amalgamation property (APK).

\section{Interpolation for tabular logics from $N E X T(\mathbf{K B} \cdot \mathbf{A l t}(\mathbf{2}))$}

Let us recall that there are only two extensions of KTB.Alt $(\mathbf{3})$ with the interpolation property. In contrast, we shall prove that there is infinitely many tabular logics in $N E X T(\mathbf{K B} \cdot \mathbf{A l t}(\mathbf{2}))$ with interpolation. Let us remind that the logic KB.Alt(2) is determined by the class of symmetric Kripke frames (we allow irreflexivity) where each point sees at most two points (including itself). Hence if $x$ is irreflexive, then it may see two distinct points; if it is reflexive then it can see only one distinct point. The Kripke frames for KB.Alt $(2)$ may be chains (possibly infinite) with irreflexive inside points. See Figure 2 where eight-point $K B$.Alt(2)-frames are presented.

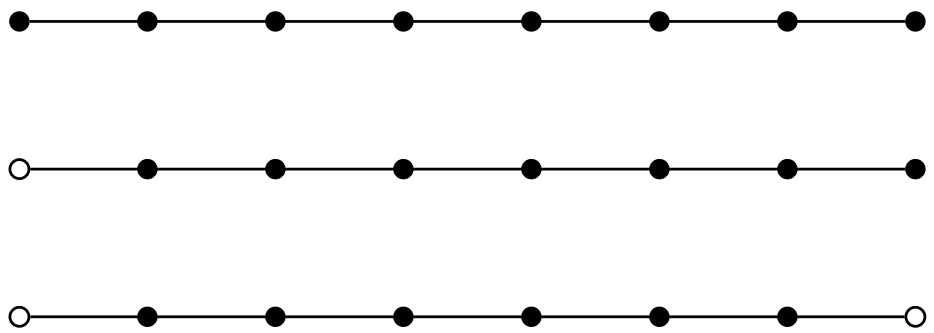

Fig. 2 .

In the class of $K B$.Alt(2)-frames we shall distinguish finite chain frames with one of the end point being reflexive. We call them almost irreflexive chains. Formally: 
DEFINITION 5. The almost irreflexive chain frame $\mathfrak{C h}_{m}$ is defined as follows: $\mathfrak{C h}_{m}:=\langle\{1,2, \ldots, m\}, R\rangle, m \geq 1$ and

$$
R:=\{(i, j), i, j=1,2, \ldots, n,|i-j|=1\} \cup\{(1,1)\} .
$$

On may see that $\mathfrak{C h}_{1}=0$.

Below, we shall describe the p-morphisms between almost irreflexive chain frames $\left\{\mathfrak{C h}_{m}, m \geq 1\right\}$.

Lemma 3. There is a unique p-morphism from $\mathfrak{C h}_{3 m-1}$ onto $\mathfrak{C h}_{m}$ for any $m \geq 1$.

Proof: Let $\mathfrak{C h}_{m}=\langle\{1,2, \ldots, m\}, R\rangle$ and $\mathfrak{C h}_{3 m-1}=\langle\{1,2, \ldots, 3 m-1\}, S\rangle$. Point 1 is the only reflexive point in both $\mathfrak{C h}_{m}$ and $\mathfrak{C h}_{3 m-1}$. We define:

$$
\begin{aligned}
f(1) & =1, f(2)=2, \quad f(3)=3, \ldots f(m)=m, \\
f(m+1) & =m-1, \quad f(m+2)=m-2, \ldots f(2 m-1)=1 \\
f(2 m) & =1, \quad f(2 m+1)=2, \quad f(2 m+2)=3, \ldots f(3 m-1)=m .
\end{aligned}
$$

One may easily see that $f$ is indeed a p-morphism from $\mathfrak{C h}_{3 m-1}$ onto $\mathfrak{C h}_{m}$. For $m=3$ the p-morphism $f$ is presented in Figure 3.

We shall explain why the p-morphism $f$ is a unique one. First, from the condition (p2) of Definition 3 we know that the reflexive point 1 from $\mathfrak{C h}_{3 m-1}$ must be mapped onto 1 from $\mathfrak{C h}_{m}$.

Second, we prove that any function $f$ gluing two neighboring irreflexive points in $\mathfrak{C h}_{3 m-1}$ and mapping them onto some irreflexive point from $\mathfrak{C h}_{m}$ is not a p-morphism. Suppose, on the contrary, that $i S i+1$ for $1<i \leq$ $3 m-4, m \geq 2$ and $f(i)=f(i+1)=j, j \neq 1$. Then we get $j R j$ and it is a contradiction. Let us notice that gluing two neighboring irreflexive points by a p-morphism causes reflexivity of the point they are mapped onto. Similarly, one may prove that any function gluing more than two neighboring points in $\mathfrak{C h}_{3 m-1}$ and mapping them onto any point from $\mathfrak{C h}_{m}$ is not a p-morphism.

Then we see that the p-morphism from $\mathfrak{C h}_{3 m-1}$ onto $\mathfrak{C h}_{m}$ has to start at 1 and then has to move along $\mathfrak{C h}_{3 m-1}$ with a short (1-step) stop at the final point $m$ and a long (2-step) stop at 1 and further until $m$. The point $m$ is the final point of the whole journey. 


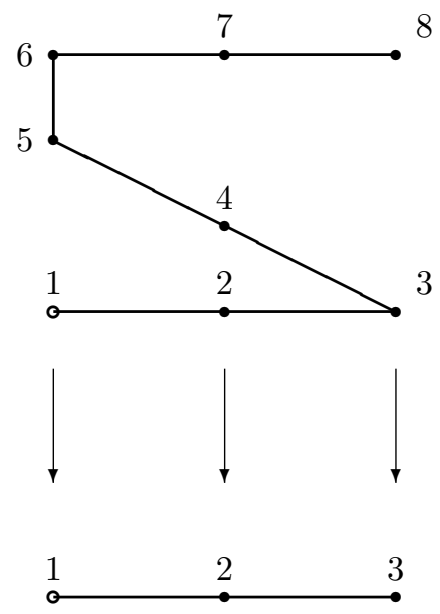

Fig. 3.

One may notice that the frame $\mathfrak{C h}_{3 m-1}$ is the minimal almost irreflexive chain frame which can be non-trivially reducible onto $\mathfrak{C h}_{m}, m \geq 1$.

We may generalize the above lemma by proving the existence of a suitable p-morphism from $\mathfrak{C h}_{m+(2 m-1) k}$ onto $\mathfrak{C h}_{m}$, for $k \geq 1$. Then the frame with $m+(2 m-1) k$ points is folded $k$-times. See Figure 4 for $m=3$. LEMMA 4. There is a unique p-morphism from $\mathfrak{C h}_{m+(2 m-1) k}$ onto $\mathfrak{C h}_{m}$ for any $m, k \geq 1$.

Proof: Obvious.

The above lemma may be strengthened to the following equivalence.

TheOREM 3. Let $n \geq 2$. Then $\mathfrak{C h}_{n}$ is reducible onto $\mathfrak{F}$ iff $\mathfrak{F}=\mathfrak{C h}_{m}$ for some $k \geq 0$ and $m \geq 1$ such that $n=m+(2 m-1) k$.

Proof: $(\Leftarrow)$ See Lemma 4 .

$$
(\Rightarrow)
$$

Case $1 . \mathfrak{F}=\circ$. It means that $\mathfrak{F}=\mathfrak{C h}_{1}$. It is easy to observe that any almost irreflexive frame $\mathfrak{C h}_{n}$ is reducible onto $\mathfrak{C h}_{1}$. Each natural number $n$ can be written as $n:=1+k, k \geq 0$.

Case 2. $\mathfrak{F} \neq$ o. Let us observe that $\mathfrak{F}$ as a reduct of some $\mathfrak{C h}_{n}, n \geq 2$ must be a $K B$.Alt(2)-frame. It is because any p-morphism can not move out 

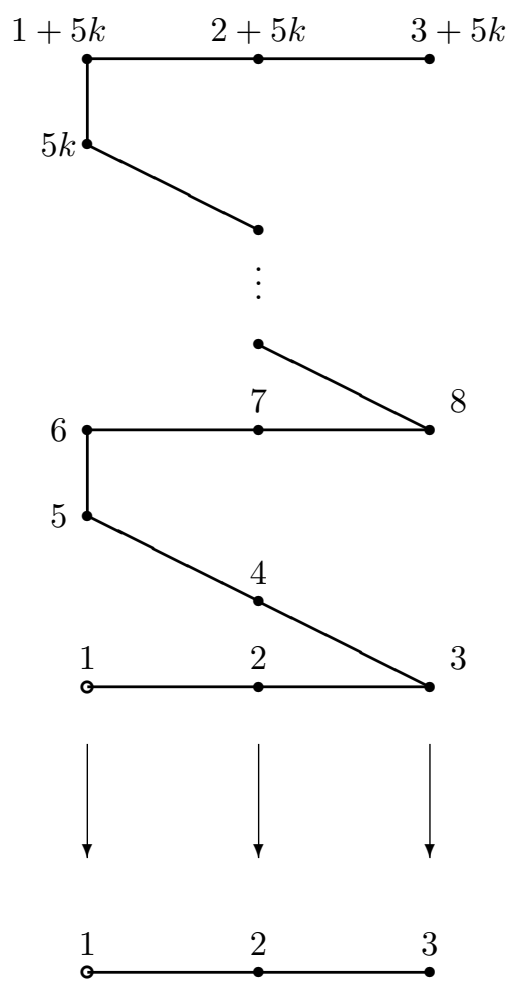

Fig. 4 .

outside the class of $K B$.Alt(2)-frames. We shall prove that $\mathfrak{F}$ is an almost irreflexive chain frame.

Let $\mathfrak{C h}_{n}=\left\langle\left\{x_{1}, x_{2}, \ldots, x_{n}\right\}, R\right\rangle$ with $x_{1}$ being the reflexive point and $n \geq 3$. From the condition (p2) of Definition 3 we see that $x_{1}$ must be mapped onto a reflexive point. Then $\mathfrak{F}$ has to include at least one reflexive point (and it must be the end point). Let $\mathfrak{F}=\left\langle\left\{y_{1}, y_{2}, \ldots, y_{m}\right\}, S\right\rangle$ and point $y_{1}$ is reflexive. Because $\mathfrak{F} \neq \circ$ then $m \geq 2$. We show that the point $y_{m}$ must be irreflexive. Suppose, on the contrary, that $y_{m}$ for $m \geq 2$ is a reflexive point, $f\left(x_{1}\right)=y_{1}$, and $f\left(x_{n}\right)=y_{m}$. Then $f\left(x_{n}\right) S y_{m}$ and from (p3) of 
Definition 3 we need another point, say $x_{k}$ in $\mathfrak{C h}_{n}$ such that $x_{k} R x_{n}$ to map it onto $y_{m}$. Hence $k=n-1$ and $f\left(x_{n-1}\right)=y_{m}$. Further we have two possibilities for the point $x_{n-2}: f\left(x_{n-2}\right)=y_{m-1}$ or $f\left(x_{n-2}\right)=y_{m}$. But in both these cases we get $f\left(x_{n}\right) S y_{m-1}$ and it means that there should exist an element $x_{s} R x_{n}$ such that $f\left(x_{s}\right)=y_{m-1}$. So, we must take $s=n-1$. This leads to a contradiction because we have already defined: $f\left(x_{n-1}\right)=y_{m}$. Analogously, one may prove that the irreflexive point $x_{n}$ from $\mathfrak{C h}_{n}$ can not be mapped onto the reflexive point $y_{1}$ from $\mathfrak{F}$. Hence, indeed $\mathfrak{F}=\mathfrak{C h}_{m}$ for some $m<n$.

Further, we shall prove the existence of $k \geq 0$ and $m \geq 1$ such that $n=m+(2 m-1) k$.

Defining the needed p-morphism we have to map $x_{1}$ onto $y_{1}$. Then the mapping goes further along the $\mathfrak{C h}_{m}$, turn over at $y_{m}$. Then we need $m-1$ points $y_{m+1}, \ldots, y_{2 m-1}$ to reach again the point $x_{1}$. Because it is reflexive then we map onto it two consecutive points $x_{2 m-1}$ and $x_{2 m}$. The mapping can not stop at this point, we need again $m-1$ points to reach the point $y_{m}$ in $\mathfrak{C h}_{m}$. So, we see that in such a case $n=m+(m-1)+1+(m-1)=3 m-1$. But if $n>3 m-1$ then the whole journey repeats and we need another $(m-1)+1+(m-1)$ points. We would get $n=3 m-1+(m-1)+1+(m-1)=$ $5 m-2$ and we see that after two 'full turns' $k=2$ and $n=m+(2 m-1) 2$. If $n>m+(2 m-1) 2$ we have to continue the mapping and so on.

Depending on the number $k$ of 'full turns' we need $m+(2 m-1) k$ points in the larger frame.

Let $\mathbb{P}:=\left\{n \in \mathbb{N}: \exists_{p \geq 3} n=\frac{p+1}{2} \& p\right.$ - prime $\}$.

LEMMA 5. There is infinitely many distinct logics in NEXT(KB.Alt (2)) which are $L\left(\mathfrak{C h}_{n}\right), n \in \mathbb{P}$.

Proof: Let us take the sequence $p_{1}, p_{2}, \ldots$ of the consecutive prime numbers larger than 2 and consider the following almost irreducible frames $\mathfrak{C h}_{2}, \mathfrak{C h}_{3}, \mathfrak{C h}_{4}, \mathfrak{C h}_{6}, \mathfrak{C h}_{7}, \mathfrak{C h}_{9}, \mathfrak{C h}_{10}, \mathfrak{C h}_{12} \ldots$, indexed with numbers from $\mathbb{P}$. We shall prove that each of them is reducible onto o, only. Suppose, on the contrary, that there is some $\mathfrak{C h}_{\frac{p+1}{2}}$ reducible onto some $\mathfrak{C h}_{m}, m \geq 2$ (see Theorem 3 ). We get that there must exist $k \geq 1$ such that it holds: $\frac{p+1}{2}=m+(2 m-1) k$. After simple calculation we get:

$$
\begin{aligned}
& p+1=2 m+(2 m-1) 2 k, \\
& p=2 m-1+(2 m-1) 2 k, \\
& p=(2 m-1)(2 k+1) .
\end{aligned}
$$


But in the last line we get a contradiction since $p$ is a prime number. From the above and from Lemma 2 we conclude that $L\left(\mathfrak{C h}_{n}\right) \not \subset L\left(\mathfrak{C h}_{m}\right)$ and $L\left(\mathfrak{C h}_{m}\right) \not \subset L\left(\mathfrak{C h}_{n}\right)$ for $n \neq m, n, m \in \mathbb{P}$.

COROLlary 1. If $n \in \mathbb{P}$ then there is no other reduct of $\mathfrak{C h}_{n}$ than $\mathfrak{C h}_{1}=0$.

Let us go back to the interpolation property. We are ready to prove:

Theorem 4. All the logics $L\left(\mathfrak{C h}_{n}\right)$ with $n \in \mathbb{P}$ have (IPD).

Proof: The logic $L\left(\mathfrak{C h}_{n}\right)$ with $n \in \mathbb{P}$ is determined by the the class consisting of one almost irreflexive chain frame. After closing this class under p-morphisms we add the one point frame o. Suppose we take as $\mathfrak{F}_{1}$ and $\mathfrak{F}_{2}$ the frame $\mathfrak{C h}_{n}$ (twice) and as $\mathfrak{F}_{0}$ the frame $\circ$. The p-morphisms $f_{1}: \mathfrak{F}_{1} \rightarrow \mathfrak{F}_{0}$ and $f_{2}: \mathfrak{F}_{2} \rightarrow \mathfrak{F}_{0}$ glue all the points into $\circ$. As $\mathfrak{F}$ we have to again take $\mathfrak{C h}_{n}$. The p-morphisms $g_{1}: \mathfrak{F} \rightarrow \mathfrak{F}_{1}$ and $g_{2}: \mathfrak{F} \rightarrow \mathfrak{F}_{2}$ are isomorphisms. Since $\mathfrak{F}_{0}=\circ$ then obviously $f_{1} \circ g_{1}=f_{2} \circ g_{2}$.

One may notice that other choices from $\left\{\mathfrak{C h}_{n}, \circ\right\}, n \in \mathbb{P}$ for $\mathfrak{F}_{i}, i:=$ $0,1,2$ lead to the same equalities. We see that in all these cases the classes of frames have (APK), hence the logics determined by them have (IPD), (see Theorem 2).

One may easily see that the logics $L\left(\mathfrak{C h}_{n}\right)$ with $n \in \mathbb{P}$ do not have (CIP). From Lemma 5 and Theorem 4 we get:

COROLlaRY 2. There is infinitely many tabular logics with (IPD) in $N E X T(\mathbf{K B} \cdot \operatorname{Alt}(\mathbf{2}))$.

One may ask question about interpolation of the other logics than $L\left(\mathfrak{C h}_{n}\right)$ with $n \in \mathbb{P}$. They are the following: $L\left(\mathfrak{C h}_{5}\right), L\left(\mathfrak{C h}_{8}\right), L\left(\mathfrak{C h}_{11}\right), \ldots$. The answer is a positive one.

Lemma 6. The logics $L\left(\mathfrak{C h}_{n}\right)$ with $n \in \mathbb{N} \backslash \mathbb{P}$ have (IPD).

Proof: Let $n \in \mathbb{N} \backslash \mathbb{P}$ and let $\mathfrak{C h}_{n} \in \mathcal{K}$. First, we closed $\mathcal{K}$ under p-morphisms. From Theorem 3 we know that if $\mathfrak{C h}_{n}$ is reducible then $n=m+(2 m-1) k$ for some $k \geq 0$ and $m \geq 1$. The reduct of $\mathfrak{C h}_{n}$ is then the frame $\mathfrak{C h}_{m}$. Because $n \in \mathbb{N} \backslash \mathbb{P}$ then $m \geq 2$.

Case 1. Suppose that there is not another $m^{\prime} \geq 2$ such that $n=$ $m^{\prime}+\left(2 m^{\prime}-1\right) k^{\prime}$ for some $k^{\prime} \geq 0$ (and $\left.m^{\prime} \geq 2\right)$. Then $\mathcal{K}=\left\{\mathfrak{C h}_{n}, \mathfrak{C h}_{m}, \circ\right\}$. For any choices of frames from $\mathcal{K}$ for $\mathfrak{F}_{1}, \mathfrak{F}_{2}, \mathfrak{F}_{0}$ and $\mathfrak{F}(\mathrm{APK})$ holds.

Such a situation takes place, i.e. for $\mathfrak{C h}_{5}$ which is reducible onto $\mathfrak{C h}_{2}$. In turn $\mathfrak{C h}_{2}$ is reducible only onto $\circ$. 
Case 2. Suppose that there are another pair of numbers $k^{\prime} \geq 0$ and $m^{\prime} \geq 1$ such that $n=m^{\prime}+\left(2 m^{\prime}-1\right) k^{\prime}\left(m^{\prime} \neq m, m^{\prime} \geq 2\right)$ and $\mathfrak{C h}_{n}$ is reducible also onto the frame $\mathfrak{C h}_{m^{\prime}}$.

Sub-case 2a. Suppose that the common reduct of $\mathfrak{C h}_{m}$ and $\mathfrak{C h}_{m^{\prime}}$ is only $\mathfrak{C h}_{1}=$ o. Then $\mathcal{K}=\left\{\mathfrak{C h}_{n}, \mathfrak{C h}_{m}, \mathfrak{C h}_{m^{\prime}}, \circ\right\}$. To show that (APK) holds we have to choose as the frames $\mathfrak{F}_{1}$ and $\mathfrak{F}_{2}$ some frames from $\mathcal{K}$. The less trivial choice is: $\mathfrak{F}_{1}=\mathfrak{C h}_{m}$ and $\mathfrak{F}_{2}=\mathfrak{C h}_{m^{\prime}}$. Then obviously $\mathfrak{F}_{0}=0$. Then, we have to select: $\mathfrak{F}=\mathfrak{C h}_{n}$. Because $\mathfrak{F}_{0}=0$ then (APK) holds. For other choices (APK) holds as well.

Such a situation takes place, i.e. for $\mathfrak{C h}_{8}$ which is reducible onto $\mathfrak{C h}_{3}$ and $\mathfrak{C h}_{2}$. In turn $\mathfrak{C h}_{3}$ and $\mathfrak{C h}_{2}$ are both reducible only onto o.

Sub-case 2 b. Suppose that the common reduct of $\mathfrak{C h}_{m}$ and $\mathfrak{C h}_{m^{\prime}}$ is some $\mathfrak{C h}_{k}, k \neq 1$. Again the less trivial choice is: $\mathfrak{F}_{1}=\mathfrak{C h}_{m}, \mathfrak{F}_{2}=\mathfrak{C h}_{m^{\prime}}$ and $\mathfrak{F}_{0}=\mathfrak{C h}_{k}$. Then we select: $\mathfrak{F}=\mathfrak{C h}_{n}$. We denote the existing reductions as follows:

$$
\begin{aligned}
& f_{1}: \mathfrak{C h}_{m} \rightarrow \mathfrak{C h}_{k}, \quad f_{2}: \mathfrak{C h}_{m^{\prime}} \rightarrow \mathfrak{C h}_{k}, \\
& g_{1}: \mathfrak{C h}_{n} \rightarrow \mathfrak{C h}_{m}, \quad g_{2}: \mathfrak{C h}_{n} \rightarrow \mathfrak{C h}_{m^{\prime}},
\end{aligned}
$$

and all the functions: $f_{1}, f_{2}, g_{1}, g_{2}$ are unique. Then their superpositions $f_{1} \circ g_{1}$ and $f_{2} \circ g_{2}$ are unique and $f_{1} \circ g_{1}=f_{2} \circ g_{2}$. For other choices of frames (APK) holds as well.

Such a situation takes place, i.e. for $\mathfrak{C h}_{53}$ which is reducible onto $\mathfrak{C h}_{8}$ and $\mathfrak{C h}_{11}$. In turn $\mathfrak{C h}_{8}$ and $\mathfrak{C h}_{11}$ are both reducible only onto $\mathfrak{C h}_{2}$.

We allow here that $k=m$ or $k=m^{\prime}$. The example is the following: $n=14, m=5, m^{\prime}=k=2$. Then $f_{2}$ is isomorphism.

Corollary 3. All the logics $L\left(\mathfrak{C h}_{n}\right)$ with $n \in \mathbb{N}$ have (IPD).

We proved that there exist countably infinitely many logics with (IPD) in $N E X T($ KB.Alt $(\mathbf{2}))$. It is quite opposite than in $N E X T($ KTB.Alt $(\mathbf{3}))$ where there are only two logics with interpolation.

It seems that reflexivity of Kripke frames negatively affects the amalgamation property (and hence interpolation for the determined logics).

The following problems are left open:

Problem 1. Whether the logic KB.Alt(2) has interpolation?

PROBLEM 2. Is there a tabular logic (different from $L(\circ)$ or $L(\circ--\circ)$ ) with interpolation in $N E X T(\mathbf{K T B}$.Alt(n)) with $n \geq 4$ ? 


\section{References}

[1] A. Chagrov, M. Zakharyaschev, Modal Logic, Oxford Logic Guides 35, (1997).

[2] J. Czelakowski, Logical matrices and the amalgamation property, Studia Logica 41 (4), (1981), pp. 329-341.

[3] D. M. Gabbay, Craig's interpolation theorem for modal logics, [in:] W. Hodges (ed.), Proceedings of logic conference, London 1970, Vol. 255 of Lecture Notes in Mathematics, Springer-Verlag, Berlin, (1972), pp. 111-127.

[4] Z. Kostrzycka, On interpolation and Halldén-completeness in $N E X T(K T B)$, Bulletin of the Section of Logic Vol. 41:1/2 (2012), pp. 23-32.

[5] Z. Kostrzycka, Interpolation in normal extensions of the Brouwer logic, Bulletin of the Section of Logic, Vol. 45:3/4 (2016), pp. 1-15.

[6] L. Maksimowa, Interpolation theorems in modal logics and amalgamated varieties of topoboolean algebras, (in Russian), Algebra i Logika, Vol. 18 (1979), pp. 556-586.

[7] Y. Miyazaki, Normal modal logics containing KTB with some finiteness conditions, Advances in Modal Logic 5, pp. 171-190, DOI: 10.1007/s11225007-9056-7.

University of Technology

ul. Sosnkowskiego 31, 45-272 Opole

Poland

e-mail: z.kostrzycka@po.opole.pl 